\title{
Using Eulerlets to model steady uniform flow past a circular cylinder
}

\author{
E. Chadwick ${ }^{\mathrm{a}}$, J. Christian and K. Chalasani
}

${ }^{a}$ University of Salford, Salford, UK

\section{ARTICLE HISTORY}

Compiled September 11, 2018

\begin{abstract}
Consider uniform, steady flow past a circular cylinder at Reynolds numbers 26, 36 and 40 before the flow becomes unsteady. Model the flow by using eulerlets, new Green's functions for Euler flow. This is the first time this eulerlet model has been used, introduced at the recent BETEQ 2017 International Conference. In addition, the far-field is also obtained by matching with oseenlets. Unlike existing Euler flow representations, the drag, wake eddies and far-field wake profile are all captured, and compare favourably with experiment.
\end{abstract}

\section{KEYWORDS}

Eulerlets, Oseenlets

\section{Introduction}

The novelty in the paper is to demonstrate the use of two-dimensional eulerlets in modelling bluff body flows. Eulerlets are the Green's function solution to the Euler equations given for two-dimensional flow by Chadwick and Kapoulas in (Chadwick, 2013, 2015; Chadwick and Kapoulas, 2015). Eulerlet models are different from usual Euler representations in that they model a wake, crucial for bluff body flows. Existing Euler flow representations for separated flow use free streamline theory (Goldstein, 1960), but it is evident that the flow physics and predictions from these theories are poor (Kiya and Arie, 1977). In the present paper, two-dimensional flow past a circular cylinder is considered, and also the far-field is modelled by matching the near-field eulerlets to the corresponding far-field oseenlets.

\section{The two-dimensional eulerlets}

The two-dimensional eulerlets are given by

$$
\begin{aligned}
u_{i}^{(1)} & =\frac{1}{2 \pi}[\ln r]_{, i}-H\left(x_{1}\right) \delta\left(x_{2}\right) \delta_{i 1} \\
u_{i}^{(2)} & =\frac{1}{2 \pi} \epsilon_{i j 3}[\ln r]_{, i}=\frac{1}{2 \pi}[-\theta]_{, i}
\end{aligned}
$$




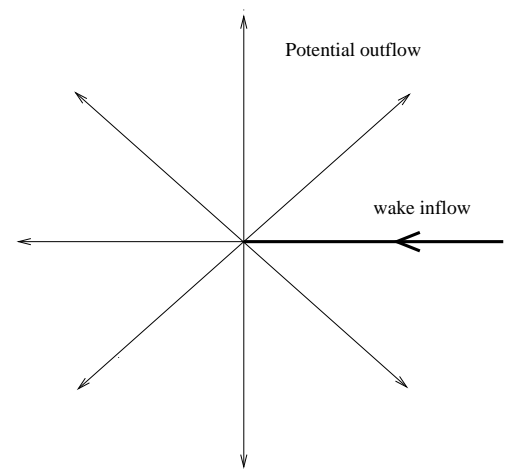

Figure 1. Streamlines for the drag eulerlet

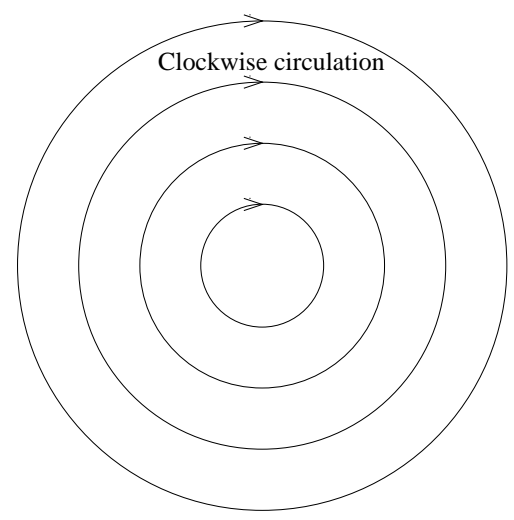

Figure 2. Streamlines for the two-dimensional lift eulerlet

where $u_{i}^{(k)}$ is generated by a unit point force at the origin in the $k$ th direction, for the Cartesian co-ordinates $x_{i}$ in Einstein tensor notation such that a comma denotes a derivative. In two-dimensions the radius and polar angle are given by $r$ and $\theta$ respectively, and in three-dimensions the radius is given by $R$. The heaviside function is denoted by $H\left(x_{1}\right)$ where $H\left(x_{1}\right)=0$ for $x_{1}<0$ and $H\left(x_{1}\right)=1$ for $x_{1}>0$ and picks out the wake. The Dirac delta function is denoted by $\delta$ with the additional property that its derivative is zero (We note that this does not contradict the delta function property $x \frac{\partial \delta(x)}{\partial x}=-\delta(x)$, which still holds, and so this is is an additional property to those that already apply.). Kronecker delta is given by $\delta_{i j}$ such that $\delta_{i j}=1$ for $i=j$ and $\delta_{i j}=0$ for $i \neq j$, and the Levi-Civita symbol $\epsilon_{i j k}$ is given by $\epsilon_{i j k}=1$ for $(i, j, k)=(1,2,3),(2,3,1),(3,1,2), \epsilon_{i j k}=-1$ for $(i, j, k)=(1,3,2),(2,1,3),(3,2,1)$, $\epsilon_{i j k}=0$ otherwise.

These eulerlets are represented pictorially in figures 1 and 2 .

\section{Separated flow past a circular cylinder}

Consider modelling separated rather than attached flow past a circular cylinder of normalised radius 1 centred at the orgin by a distribution of eulerlets over the cylinder boundary producing a wake distribution. 
The fluid velocity component $u_{i}$ is represented by two parts, a velocity potential $\phi,_{i}$ and a wake velocity $w_{i}$ such that $u_{i}=\phi_{, i}+w_{i}$. The potential is given in terms of the potential parts of the eulerlets which can then be represented by a harmonic expansion originating from the origin such that

$$
\phi=\frac{D}{2 \pi} \ln r+\sum_{n=1}^{\infty} a_{n} \frac{\cos n \theta}{r^{n}}
$$

for coefficient $D$ related to the drag, and for some unknown coefficients $a_{n}$, from either use of the Taylor series expansion or complex potential theory. The wake velocity is given in terms of a distribution of the wake velocity of the drag eulerlets over the cylinder boundary, the terms of each wake eulerlet lying on the infinite half line aligned to the $x_{1}$-axis. So, this can be represented as originating from a distribution along the line $-1 \leq x_{2} \leq 1, x_{1}=0$ such that

$$
\begin{aligned}
w_{i}(\mathbf{x}) & =\int_{-1}^{1} d\left(x_{2}^{\prime}\right) w_{i}^{(1)}\left(\mathbf{x}-\mathbf{x}^{\prime}\right) d x_{2}^{\prime} \\
& =-\int_{-1}^{1} d\left(x_{2}^{\prime}\right) H\left(x_{1}\right) \delta\left(x_{2}-x_{2}^{\prime}\right) \delta_{i 1} d x_{2}^{\prime}
\end{aligned}
$$

from (1). Therefore the derivative of $w_{i}$ in the $x_{2}$ direction, and so the wake vorticity, is zero as the derivative of the Dirac delta function is zero. So (3) represents an Euler slip wake, where fluid slips past each other in layers. The total Euler drag is the wake inflow given by $D=-\int_{\partial \Sigma} w_{1} d \partial \Sigma=\int_{-1}^{1} d\left(x_{2}\right) d x_{2}$, and from (1) the outflow is $\int_{\partial \Sigma} \phi,{ }_{i} n_{i} d \partial \Sigma=D$, and the inflow is $\int_{\partial \Sigma} w_{i} n_{i} d \partial \Sigma=-D$.

Euler flow and the eulerlet theory assume a vanishingly thin boundary layer of thickness $\delta=0(1 / \sqrt{R} e) \rightarrow 0$, where ' 0 ' means 'of the order of' and $R e$ is the Reynolds number $R e=\rho U l / \mu$ for some near-field length dimension $l$, density $\rho$, free stream velocity $U$ and coefficient of viscosity $\mu$. However, for these problems the Reynolds number such as $R e=20$ is so low that the boundary layer thickness $\delta=1 / \sqrt{2} 0$ is sizeable. So this is a significant approximation which will signficantly limit the accuracy of the model.

\subsection{Euler drag profile for cosine distribution}

Consider a cosine shaped profile for the drag eulerlet distribution such that

$$
d\left(x_{2}\right)=\frac{D}{2}\left(1+\cos \left(\pi x_{2}\right)\right)
$$

for $\left|x_{2}\right| \leq 1$, and zero otherwise. Then the Euler drag is $D=\int_{-1}^{1} d\left(x_{2}\right) d x_{2}=\int_{-1}^{1} \frac{D}{2}(1+$ $\left.\cos \left(\pi x_{2}\right)\right) d x_{2}=D$. The cosine distribution is chosen to mimic the form of the cosinelike far-field wake. 


\subsection{Boundary condition for cosine distribution}

Assuming a boundary layer of negligible thickness over the body, then the impermeability condition holds for the Euler velocity

$$
\begin{aligned}
u_{i}^{\dagger} n_{i} & =\left.0\right|_{r=1} \\
\left(\delta_{i 1}+\phi,_{i}-H\left(x_{1}\right) d\left(x_{2}\right) \delta_{i 1}\right) n_{i} & =\left.0\right|_{r=1} \\
n_{1}+\phi,{ }_{i} n_{i}-H\left(x_{1}\right) d\left(x_{2}\right) n_{1} & =\left.0\right|_{r=1} .
\end{aligned}
$$

Substituting in for $\left(n_{1}, n_{2}\right)=(\cos \theta, \sin \theta)$ and the expression for the potential (2) then gives for $r=1, x_{1}<0$ :

$$
\cos \theta+\frac{D}{2 \pi}-\sum_{n=1}^{\infty} n a_{n} \cos n \theta=0
$$

and for $r=1, x_{1}>0$ :

$$
\cos \theta+\frac{D}{2 \pi}-\sum_{n=1}^{\infty} n a_{n} \cos n \theta-\frac{D}{2}(1+\cos (\pi \sin \theta) \cos \theta)=0
$$

It is noticed that at least one coefficient $a_{n}$ does not linearly depend upon the drag. However, from Abramowitz and Stegun (Abramowitz and Stegun, 1972) it can be shown that

$$
\cos (\pi \sin \theta) \cos \theta=\sum_{n=0}^{\infty} \frac{2(2 n+1)}{\pi} J_{2 n+1}(\pi) \cos [(2 n+1) \theta]
$$

from 9.1.42 p361, 4.3.32 p72 and 9.1.27 p361, where $J_{n}$ is the Bessel function of the first kind of order $n$. So for $r=1, x_{1}>0$ :

$$
\cos \theta+\frac{D}{2 \pi}-\sum_{n=1}^{\infty} n a_{n} \cos n \theta-\frac{D}{\pi} \sum_{n=0}^{\infty}(2 n+1) J_{2 n+1}(\pi) \cos (2 n+1) \theta-\frac{D}{2} \cos \theta=0 .
$$

\section{Fourier analysis to determine the coefficients for cosine distribution}

In this section, we apply Fourier analysis to determine the coefficients $a_{n}$. The boundary condition is

$$
\begin{aligned}
& \int_{0}^{\pi} \cos m \theta\left\{\cos \theta+\frac{D}{2 \pi}-\sum_{n=1}^{\infty} n a_{n} \cos n \theta\right\} \mathrm{d} \theta \\
& -\frac{D}{2} \int_{0}^{\pi / 2} \cos m \theta\left\{\cos \theta+\frac{2}{\pi} \sum_{n=0}^{\infty}(2 n+1) J_{2 n+1}(\pi) \cos ((2 n+1) \theta)\right\} \mathrm{d} \theta=0 .
\end{aligned}
$$


However, we have that

$$
\int_{0}^{\pi} \cos m \theta \cos n \theta \mathrm{d} \theta=\left\{\begin{array}{cc}
0 & m \neq \pm n \\
\pi / 2 & m= \pm n \\
\pi & m=n=0
\end{array}\right.
$$

and

$$
\int_{0}^{\pi / 2} \cos m \theta \cos [(2 n+1) \theta] \mathrm{d} \theta=\left\{\begin{array}{cll}
0 & m \text { odd, } & m \neq \pm(2 n+1) \\
\pi / 4 & m \text { odd, } & m= \pm(2 n+1) \\
\frac{(-1)^{n+m / 2}(2 n+1)}{(2 n+1)^{2}-m^{2}} & m \text { even. }
\end{array}\right.
$$

So for $m=0,(10)$ becomes

$$
\frac{D}{2}-\frac{D}{2}-\frac{D}{\pi} \sum_{n=0}^{\infty}(-1)^{n} J_{2 n+1}(\pi)=0
$$

which is true since $2 \sum_{n=0}^{\infty}(-1)^{n} J_{2 n+1}(\pi)=\sin (\pi)=0$ from Abramowitz and Stegun (Abramowitz and Stegun, 1972) 9.1.48 p361. Furthermore, substituting in for $m=1$ into (10) gives

$$
\begin{aligned}
\frac{\pi}{2} a_{1} & =\frac{\pi}{2}-\frac{\pi}{2} \frac{D}{4}-\frac{\pi}{2} \frac{D}{2 \pi} J_{1}(\pi) \\
a_{1} & =1-D / 4-(D / 2 \pi) J_{1}(\pi)
\end{aligned}
$$

for $m \geq 2$ and even giving

$$
\begin{aligned}
-\frac{\pi}{2} m a_{m} & =\frac{D}{\pi} \sum_{n=0}^{\infty} \frac{(-1)^{n+m / 2} J_{2 n+1}(\pi)}{1-(m /(2 n+1))^{2}}+\frac{D}{2}\left(\frac{(-1)^{m / 2}}{1-m^{2}}\right) \\
a_{m} & =\frac{2 D}{m \pi^{2}} \sum_{n=0}^{\infty} \frac{(-1)^{n+m / 2} J_{2 n+1}(\pi)}{(m /(2 n+1))^{2}-1}+\frac{D}{\pi m}\left(\frac{(-1)^{m / 2}}{m^{2}-1}\right)
\end{aligned}
$$

where we have used the result

$$
J_{n}(z)=((1 / 2) z)^{n} \sum_{k=0}^{\infty} \frac{\left(-(1 / 4) z^{2}\right)^{k}}{k ! \Gamma(n+k+1)}
$$

with $\Gamma(n+k+1)=(n+k)$ ! from Abramowitz and Stegun (Abramowitz and Stegun, 1972) 6.1 .6 and 9.1.10. Finally, for $m \geq 3$ and odd we get

$$
\begin{aligned}
-\frac{\pi}{2} m a_{m} & =\frac{m D}{4} J_{m}(\pi) \\
a_{m} & =-\frac{D}{2 \pi} J_{m}(\pi) .
\end{aligned}
$$




\subsection{Position and merging of near-and far-fields}

Let $L$ be the far-field length dimension, and $l$ be the near-field length dimension. From the oseenlet $e^{k x_{1}} K_{0}(k r) \approx e^{-k x_{2}^{2} / 2 x_{1}}$ in the far-field, where $k=\rho U / 2 \mu$ and $K_{0}$ is the modified Bessel function of order zero. So the wake is exited when $\eta=k l^{2} / 2 L=$ $(R e / 4)(l / L) \rightarrow \infty$ which also therefore defines the boundary between the near- and far-fields. For Reynolds number $R e=26,36$ and 40, this occurs even close to the circular cylinder as the Reynolds number is so small the far-field length $L$ is not large. However, we must also ensure that in the far-field, the Oseen velocity is a small perturbation of the uniform flow field. By inspection of the numerical results in figure $4 \mathrm{a}$ and $5 \mathrm{a}$ we see that this holds at radial distance $r=b=4$ for $R e=26$ and $r=b=6$ for $R e=40$. We note that the Oseen far-field has the effect of diffusing the wake and consequently closing the eddies downstream; if instead only Euler flow is considered, then the eddies remain open to infinity. The Oseen wake diffusion originates from the modified Bessel function in two-dimensional flow, and the eddy length is a distance of order Re downstream, as expected from experimental observation.

To get a smooth transition between Euler and Oseen flow, a merging of the two flow fields is proposed between the radial distances $r=a$ and $r=b$ and represented in the figure 3 .

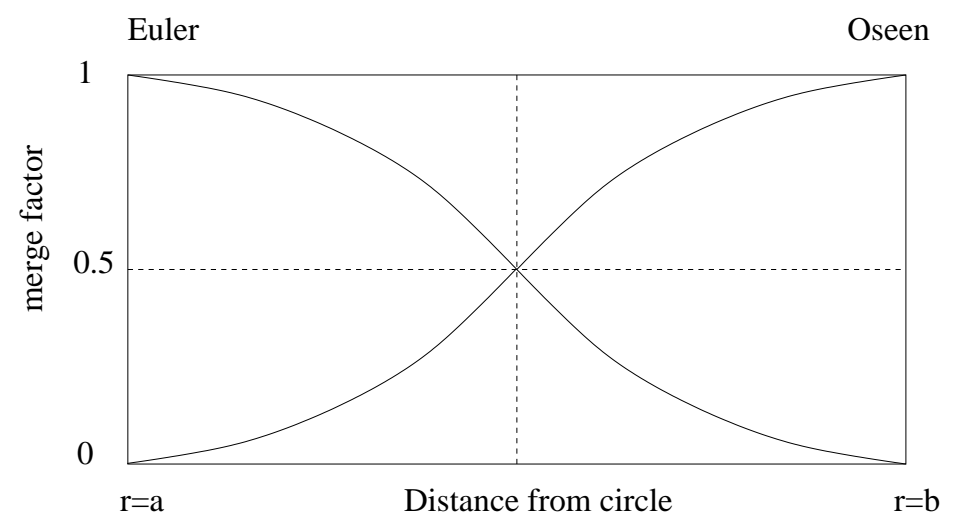

Figure 3. Merging of Euler and Oseen flow representations

The graph in the lower left hand corner is reflected about the dotted lines of symmetry to get the graphs in the other quadrants, and this graph itself has the quadratic form $y=\alpha(r-a)^{2}$, and so $\alpha=\frac{2}{(b-a)^{2}}$. In this merging region, the velocity is then given as $u_{i}^{\text {merged }}=(1-\alpha) u_{i}^{\text {Euler }}+\alpha u_{i}^{\text {Oseen }}$ where $u_{i}^{\text {Euler }}$ and $u_{i}^{\text {Oseen }}$ are the Euler and Oseen velocities respectively.

We choose $a=1$ to get the greatest distance, and therefore most gradual merging and smooth transition between the two flows. However, it is noted that changing the values of $a$ does not significantly affect either the size or the position of the eddy in the wake or the wake structure and flow itself. So the choice for $a$ is not particularly crucial to the outcome of the flow description. 


\subsection{Results}

We consider two Reynolds numbers for the flow for which there are known results, $R e=26$ with wake distribution strength $\int_{-1}^{1} d\left(x_{2}^{\prime}\right) d x_{2}^{\prime}$ of 2.0 , and $R e=40$ with wake distribution strength of 1.5. These strengths are selected from the known values of the drag by experiment at these Reynolds numbers. This produces the following flow patterns.

\subsubsection{Laminar flow Reynolds number 26}

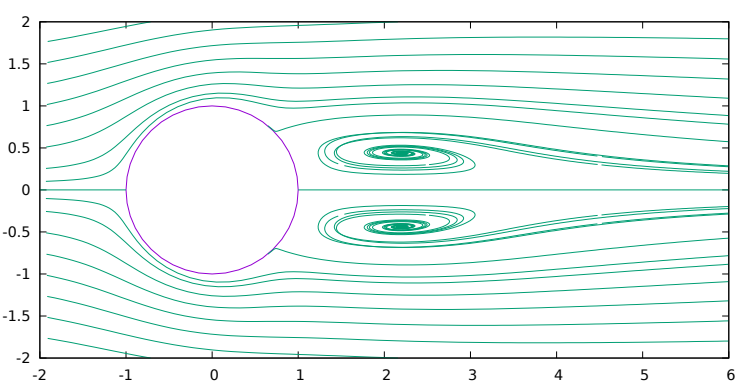

(a) Theory

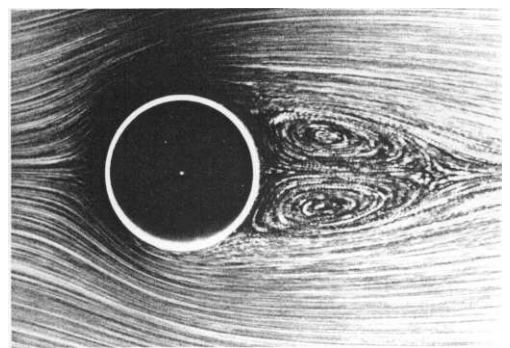

(b) Experiment: Image courtesy VanDyke Album of Fluid Motion(Dyke, 1982)

Figure 4. Flow field for $R e=26$

We note the separation point is closely matched, and the size and shape of the eddies are reproduced, although the experimental results suggest that the wake tail should be curtailed sooner.

\subsubsection{Laminar flow Reynolds number 40}

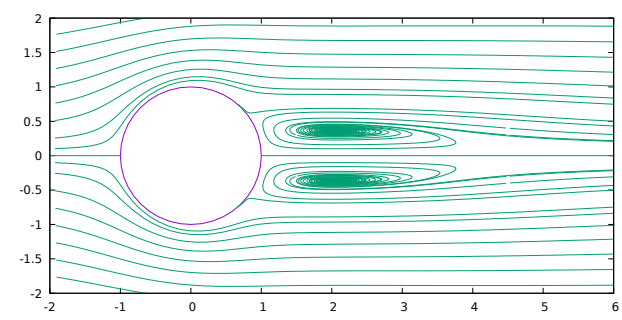

(a) Theory

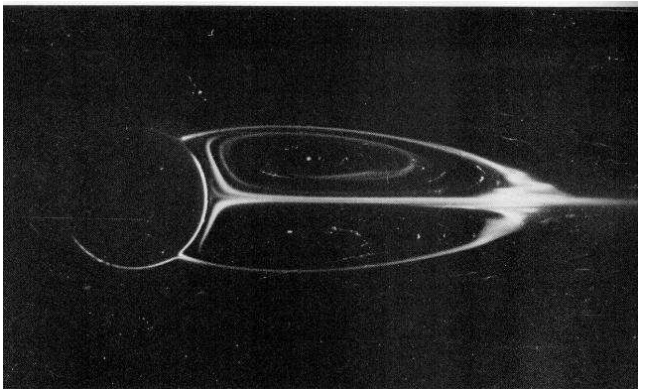

(b) Experiment: Image courtesy Coutanceau Bouard (Coutanceau and Bouard, 1949)

Figure 5. Flow field for $R e=40$

We note that the eddy position is similar and the elongation of the eddies with higher Reynolds number is captured. However, the separation point hasn't moved as far upstream as expected and the eddies are more flattened than expected from experiment. 


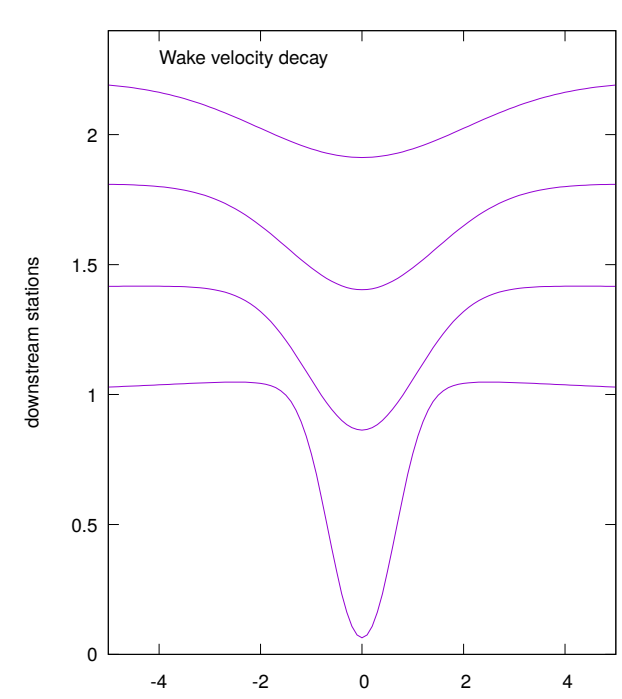

(a) Theory

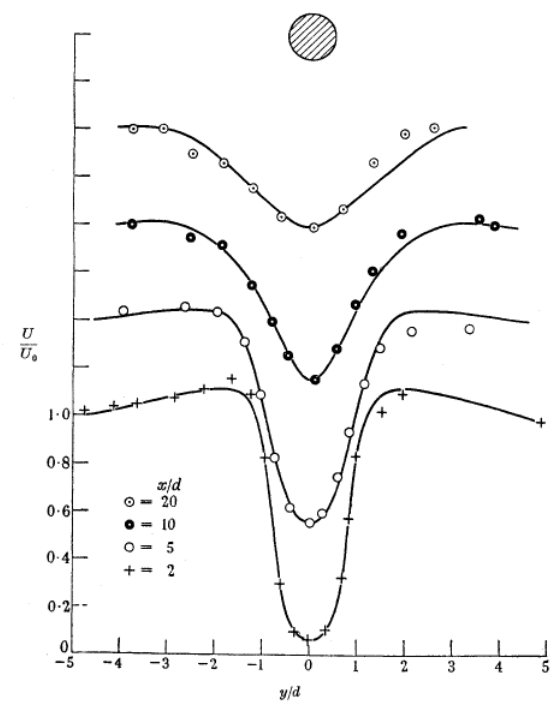

(b) Experiment

Figure 6. Far-field wake $R e=36$

We have experimental results for the far-field decay at Reynolds number $R e=36$ from Kovasnzay (Kovasznay, 1949). We note that the trend in diffusion is captured, and general wake profile is captured. However, the rise at the lip of the wake is only partially captured, see for example (Tordella and M.Belan, 2003).

\section{Conclusion}

Accuracy is limited because at a Reynolds number of 40 steady flow breaks down. Even so, the streamlines compare well with flow visualisation, and the far-field velocity distribution compares well to experiment; All the essential flow physics and the farfield flow compares well to experiment.

It is noted that there are always two unknowns in the calculations: (1) the strength of the wake distribution D and (2) the near-field boundary length. Both of these are predetermined by comparing the results with the experimental data for any particular Reynolds number. This is straightforward in the case of known experimental test problems, but presents a challenge for extending the presented approach to other geometries and a wider range of Reynolds number. One possible approach to overcome this would be to use empirical correlation. From existing experimental results for particular geometries and Reynolds numbers, empirical correlation could be use to predict the strength of the wake distribution for the particular near-field boundary length (and therefore Reynolds number). Then, from this the approach presented in the paper could be then used to determine the flow characteristics and pressure distribution. 


\section{Acknowledgement(s)}

We wish to thank Dr. T. Rahulan, Dr. O. Sugar-Gabor and Dr. D. Myring of the University of Salford Aeronautics Department, for constructive comments on the research.

\section{Disclosure statement}

There is no potential conflict of interest associated with this research.

\section{Funding}

No grants funded this work

\section{Notes on contributor(s)}

Dr. Edmund Chadwick is a Reader in Applied Mathematics and Director of the Mathematics Simulation Technology Research Team at the University of Salford. Dr. James Christian is a Lecturer in Theoretical Physics at the University of Salford. Mr. Kiran Chalasani is a Ph.D. student under the supervision of Dr. Edmund Chadwick and Dr. James Christian at the University of Salford.

\section{References}

M. Abramowitz and I.N. Stegun. Handbook of Mathematical Functions, 10th edition. Dover, New York, 1972.

E.A. Chadwick. The far-field Green's integral in Stokes flow from the boundary integral formulation. Computer Modeling in Engineering Sciences, 96(3):177-184, 2013.

E.A. Chadwick. Modelling steady flow past a two-dimensional bluff body by using eulerlets, in Advances in Boundary Element and Meshless Techniques XVI eds. V. Matic et. al. EC Ltd, Eastleigh, UK, 2015.

E.A. Chadwick and A. Kapoulas. Using eulerlets to give a boundary integral formulation in Euler flow and discussion on applications. Computer Modeling in Engineering Sciences, 102: 331-343, 2015.

M. Coutanceau and R. Bouard. Experimental Determination of the Main Features of the Viscous Flow in the Wake of a Circular Cylinder in Uniform Translation. J. Fluid Mech., 79:231-256, 1949.

M. Van Dyke. An Album of Fluid Motion. Parabolic Press, Stanford, 1982.

S. Goldstein. Lectures on Fluid Mechanics. Interscience, 1960.

M. Kiya and M. Arie. An inviscid bluff-body wake model which includes the far-wake displacement effect. J. Fluid Mech., 81(3):593-607, 1977.

L.S G. Kovasznay. Hot-Wire Investigation of the Wake behind Cylinders at Low Reynolds Numbers. Proc. Roy. Soc. A, 198:174-190, 1949.

D. Tordella and M.Belan. A new matched asymptotic expansion for the intermediate and far flow behind a finite body. Physics of Fluids, 15:7: 1897-1906, 2003. 